\title{
Frecuencia y factores de riesgo de trombosis venosa profunda asociada a pacientes con COVID-19
}

\section{Frequency and risk factors of deep venous thrombosis associated with COVID-19 patients}

\author{
Melvin Parada-Guzmán ${ }^{1 *}$, Miguel A. Sierra ${ }^{1}$, Sandra Olivares-Cruz ${ }^{1}$ y Carlos A. Balderrama ${ }^{2}$ \\ ${ }^{1}$ Servicio de Angiología y Cirugía Vascular, Hospital General de México, Ciudad de México; ${ }^{2}$ Servicio de Cirugía General, Hospital General \\ Dr. Aurelio Valdivieso, Oaxaca de Juárez. México
}

\begin{abstract}
Resumen
Antecedentes: La trombosis venosa profunda (TVP) es frecuente en pacientes con infección por enfermedad por coronavirus 2019 (COVID-19), caracterizada por respuesta inflamatoria que conduce a manifestaciones graves y puede considerarse un factor de riesgo de trombosis. Objetivo: Evaluar la frecuencia de factores de riesgo de TVP en pacientes COVID-19 en el Hospital General de México. Material y métodos: Estudio retrospectivo, que consistió en la revisión de interconsultas por trombosis, analizando factores de riesgo en 83 pacientes. Resultados: El miembro pélvico izquierdo fue el más afectado en ambos grupos, encontramos un Puntaje de Wells más alto en el grupo no COVID, 75\%. En pacientes COVID positivos, la frecuencia de diabetes mellitus e inmovilidad fue 0.19 y 0.20 respectivamente. Conclusión: Los factores de riesgo asociados con mayor frecuencia a pacientes con COVID-19 y trombosis fueron cáncer, inmovilidad y obesidad, por lo que debemos establecer una profilaxis antitrombótica temprana en este grupo de pacientes.
\end{abstract}

Palabras clave: Trombosis venosa profunda. COVID-19. Factores de riesgo. Tercer nivel de atención.

\begin{abstract}
Background: Deep vein thrombosis (DVT) is common in patients with COVID-19 infection, characterized by an inflammatory response that leads to severe manifestations and can be considered a risk factor for thrombosis. Objective: To evaluate the frequency of DVT risk factors in COVID-19 patients in the Hospital General de Mexico. Material and methods: Retrospective study, which consisted on the review of interconsultations for thrombosis, analyzing risk factors in 83 patients. Results: The left lower extremity was the most commonly affected in both groups. We found a highest Wells score in non-COVID group, $75 \%$. In COVID positive patients, diabetes mellitus and inmobility frequencies were 0.19 and 0.20 respectively. Conclusion: The risk factors most frequently associated with COVID-19 patients and thrombosis were cancer, immobility and obesity, so we must establish an early antithrombotic prophylaxis in this group of patients.
\end{abstract}

Keywords: Deep vein thrombosis. COVID-19. Risk factors. Third level of care.

Correspondencia:

*Melvin Parada-Guzmán

E-mail: pardguzman1213@gmail.com

0377-4740/@ 2021 Sociedad Mexicana de Angiología y Cirugía Vascular y Endovascular, A.C. Publicado por Permanyer. Este es un artículo open access bajo la licencia CC BY-NC-ND (http://creativecommons.org/licenses/by-nc-nd/4.0/). 


\section{Introducción}

El brote de enfermedad por coronavirus (COVID-19) fue notificado por primera vez en Wuhan, China, el 31 de diciembre de 2019 y declarada por la Organización Mundial de la Salud (OMS) como pandemia el 11 de marzo de 2020. Desde entonces, los sistemas de salud han requerido adaptación para proveer atención a los pacientes infectados y continuar con el tratamiento de patologías endémicas en la población ${ }^{1-5}$.

Es altamente probable que los meses siguientes a este tercer brote por pandemia se tenga que vivir una época de "convivencia» más o menos prolongada con la COVID-19, generando el problema de la puesta en marcha de la actividad quirúrgica, teniendo un gran impacto en muchos pacientes, sobre todo los que sufren enfermedad tromboembólica venosa y enfermedad arterial periférica ${ }^{6-10}$.

La trombosis venosa profunda (TVP) es frecuente en pacientes con infección por coronavirus 2 del síndrome respiratorio agudo grave (SARS-CoV-2), causante de la COVID-19, y se caracteriza por una respuesta inflamatoria que puede conducir a manifestaciones graves $^{10-12}$

Múltiples marcadores de coagulación apoyan esto, la COVID-19 puede considerarse un factor de riesgo de trombosis; las alteraciones del endotelio, flujo y de la función plaquetaria nos conducen a trombosis venosas y arteriales ${ }^{12}$. Efectos semejantes a los de la influenza de 1918, una trombosis pulmonar generalizada, describiéndose la formación de trombos como la principal causa en estos casos ${ }^{13-15}$.

\section{Métodos}

Estudio retrospectivo, descriptivo y observacional, llevado a cabo en el Servicio de Angiología y Cirugía Vascular del Hospital General de México (HGM), de los pacientes atendidos por nuestro servicio con diagnóstico de TVP en un periodo comprendido entre el 1 de abril y el 31 agosto de 2020, a los cuales se les realizó ultrasonido en modo $\mathrm{B}$, color y Doppler pulsado, en busca de ausencia de compresibilidad venosa, defectos intraluminales y ausencia de flujo fáscico. Se analizaron factores de riesgo de un total de 83 pacientes, 23 correspondieron a pacientes con trombosis COVID positiva y 60 con trombosis COVID negativa. Se incluyeron todos los pacientes, datos demográficos y los eventos de morbilidad, excluyéndose los pacientes con expedientes incompletos.
Una vez recabados los datos en Microsoft Excel se realizó un análisis de frecuencia y se graficó. Después de haber identificado a los pacientes COVID positivos y negativos en nuestro sistema de interconsultas, revisamos los expedientes clínicos y se analizaron variables como edad, sexo, comorbilidades, factores de riesgo, complicaciones, región y segmento anatómico. La mayoría de los pacientes por los que nos interconsultaron estaban siendo manejados por servicios de urgencias, medicina interna y cirugía general hasta su manejo definitivo, ya que no contaban con requisito de manejo específico por parte de nuestro servicio según el protocolo hospitalario.

Igualmente presentamos las complicaciones más graves en este grupo de pacientes, tromboembolia pulmonar (TEP) y flegmasías, que reflejan las condiciones de suma importancia en la enfermedad tromboembólica.

Una vez recopilada y organizada toda la información con formularios de Google forms, se realizó la suma de la base de datos y se reflejó en tablas de Microsoft Excel, presentando gráficas para su análisis.

\section{Resultados}

Podemos decir que la influencia del sexo femenino en este estudio es relevante, la mayoría de nuestros pacientes fueron mujeres ( $n=51 ; 61.4 \%$ ), un total de hombres de 32 (38.5\%); edad media de 65 años; con un total de $23(27.7 \%)$ para los pacientes COVID positivos y 60 (72.2\%) COVID negativos.

De los 83 pacientes, 10 de los casos presentaron una TVP bilateral, en general 93 extremidades inferiores, fueron valoradas en este estudio para ambos grupos, $26(27.9 \%)$ extremidades en paciente COVID positivos y 67 (72\%) COVID negativos.

El miembro pélvico izquierdo y segmento iliofemoral representan la mayoría de los casos, $43(51.8 \%)$ y $67(87 \%)$ respectivamente. Con un total de 124 segmentos anatómicos, que corresponden a segmento iliofemoral 67 (54\%), femoropoplíteo 41 (33\%), vasos tibiales $13(10.4 \%)$ y vena cava $3(2.4 \%)$ (Figs. 1 y 2).

El puntaje de Wells más alto ( $>4$ puntos) para el grupo no COVID, reportando 45 (75\%), y para el grupo COVID positivo $12(52.1 \%)$ de los casos (Fig. 3, Tabla 1).

En relación con comorbilidades en pacientes con diabetes mellitus, la frecuencia para COVID positivos $6(0.19)$, neumopatías 1 (0.03), enfermedad venosa 2(0.06) e hipertensión arterial $3(0.10)$, siendo la comorbilidad más frecuente en ambos grupos la diabetes mellitus (Fig. 4). En cuanto a los factores de riesgo para COVID positivo, 


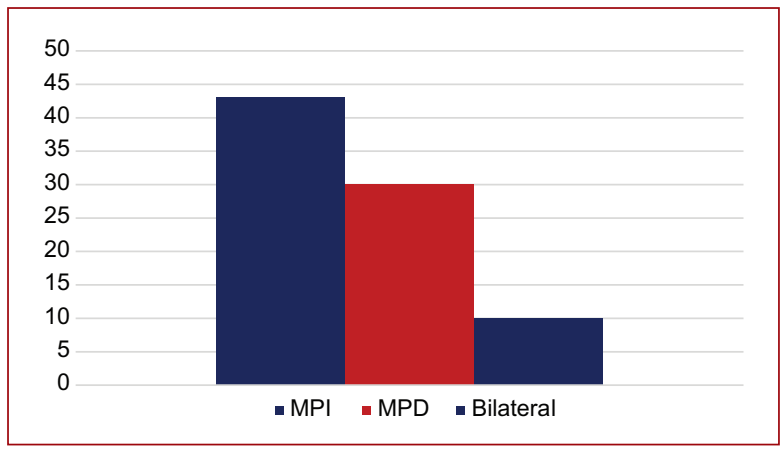

Figura 1. Miembro pélvico afectado.

MPI: miembro pélvico izquierdo; MPD: miembro pélvico derecho.

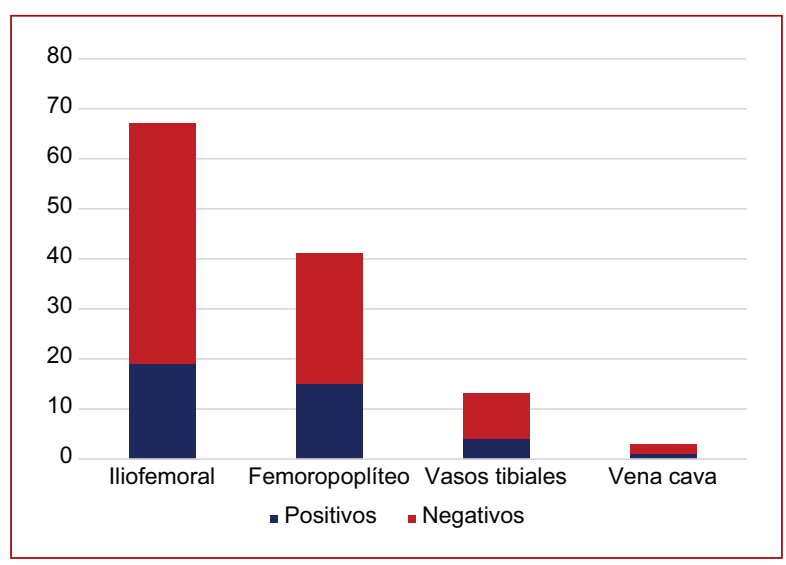

Figura 2. Segmento anatómico afectado.

la inmovilización durante la hospitalización y condiciones relacionadas con otra patología presenta una frecuencia de 15 casos (83\%), hospitalización $16(88.8 \%)$, historia de trombosis $8(44.4 \%)$, obesidad $8(44.4 \%)$ y tabaquismo $8(44.4 \%)$; la frecuencia mayor para ambos grupos en relación con factores de riesgo fue la inmovilización, representando una frecuencia total COVID positiva y negativa 0.20 y 0.17 (Fig. 5).

De los 83 pacientes estudiados se encontró que las complicaciones asociadas a la TVP fueron TEP 5 (6\%) y para la flegmasía 2 (2.4\%) (Fig. 6).

\section{Discusión}

Las infección por el SARS-CoV-2 (COVID-19) podría estar entre aquellas con mayor riesgo de sufrir complicaciones y agravamiento de enfermedades crónicas debido al extenso daño endotelial que produce estados de hipercoagulabilidad y sistema inmunitario atenuado $0^{16,17}$.

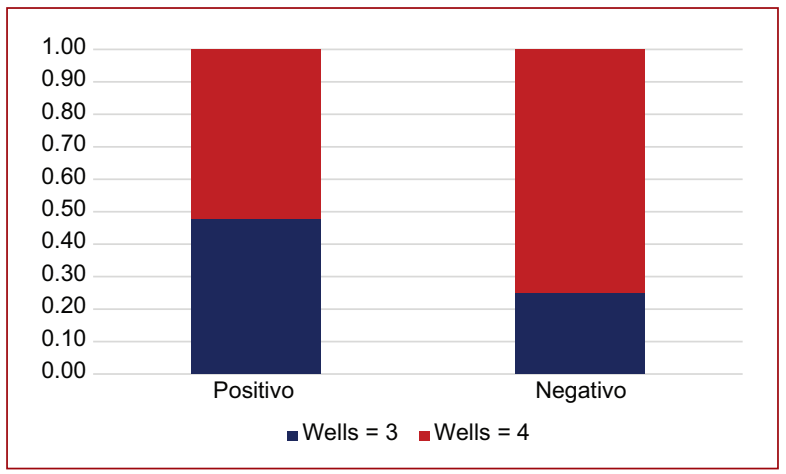

Figura 3. Frecuencia de puntaje de Wells.

Tabla 1. Escala de probabilidad clínica de trombosis venosa profunda de Wells*

\begin{tabular}{|c|c|}
\hline Característica clínica & Puntos \\
\hline $\begin{array}{l}\text { Cáncer activo (tratamiento en curso o en los últimos } \\
\text { seis meses, o paliativo) }\end{array}$ & 1 \\
\hline $\begin{array}{l}\text { Parálisis, paresia o reciente inmovilización de las } \\
\text { extremidades inferiores }\end{array}$ & 1 \\
\hline $\begin{array}{l}\text { Reciente encamamiento }>3 \text { días o cirugía } \\
\text { mayor (último mes) }\end{array}$ & 1 \\
\hline $\begin{array}{l}\text { Hipersensibilidad localizada en la distribución de las } \\
\text { venas profundas }\end{array}$ & 1 \\
\hline Edema de una extremidad & 1 \\
\hline $\begin{array}{l}\text { Hinchazón de la pantorrilla }>3 \mathrm{~cm} \text { respecto de la } \\
\text { asintomática (medida } 10 \mathrm{~cm} \text { por debajo de la } \\
\text { tuberosidad tibial) }\end{array}$ & 1 \\
\hline Edema (con fóvea) en la pierna sintomática & 1 \\
\hline Venas superficiales complementarias (no varicosas) & 1 \\
\hline Diagnóstico alternativo verosímil & -2 \\
\hline
\end{tabular}

${ }^{*}$ Alta probabilidad $\geq 3 ;$ moderada $=1-2 ;$ baja $=0$.

Un estudio anterior mostró que el $40 \%$ de los pacientes tenían un alto riesgo de desarrollar trombosis venosa, varios factores de riesgo de trombosis venosa como inmovilización, ventilación mecánica e infección podrían observarse en pacientes con COVID-19. Por lo tanto, el diagnóstico de TVP es imperativo para una clínica óptima ${ }^{18-20}$.

De acuerdo con los resultados obtenidos en nuestro estudio y comparados con lo descrito en la literatura, se presentó la frecuencia y factores de riesgo de trombosis venosa en pacientes con COVID-19. En nuestro servicio la ecografía con la utilización del Doppler demostró también que la ausencia de compresibilidad y la ausencia 


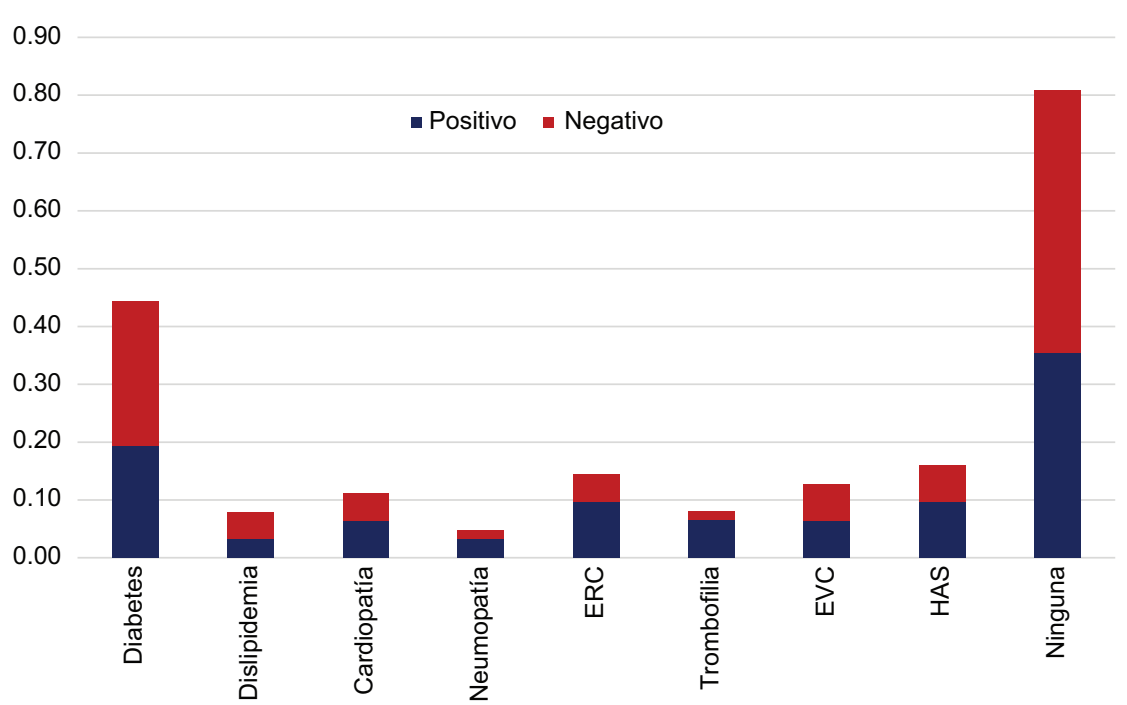

Figura 4. Frecuencia de comorbilidades en pacientes con y sin enfermedad por coronavirus 2019. ERC: enfermedad renal crónica; EVC: enfermedad cardiovascular; HAS: hipertensión arterial sistémica.

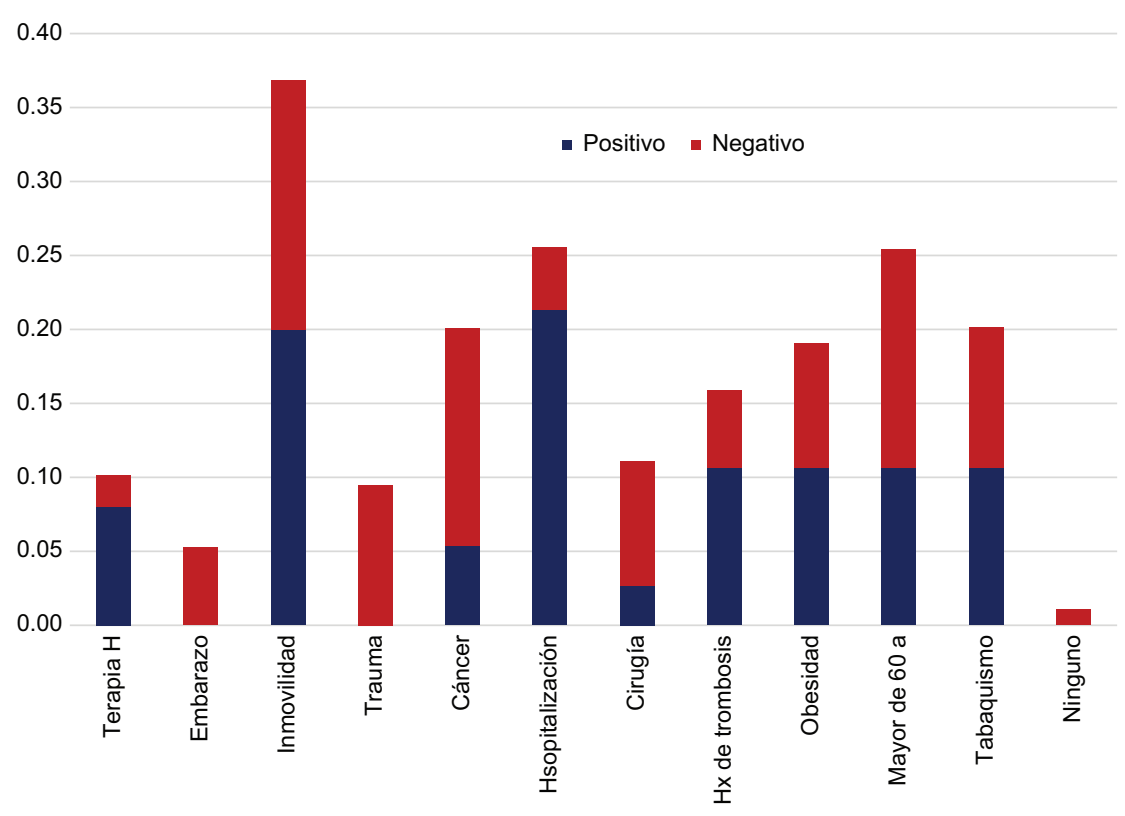

Figura 5. Frecuencia de factores de riesgo. Hx: antecedentes (historia de).

del flujo espontáneo era el hallazgo más fundamental para los residentes de angiología que practicaron el estudio y por el cual se guiaron esencialmente para llegar al diagnóstico, ya que los defectos intraluminales son difíciles de valorar en esta fase. En relación con los hallazgos ecográficos, los 83 pacientes tratados por
TVP que se sometieron a rastreo Doppler venoso de miembros inferiores dieron positivo $78(94.3 \%)$ en su primera valoración, mientras que en $5(5.7 \%)$ de nuestros pacientes no fue posible realizar estudio por las condiciones hemodinámicas durante su visita inicial, posponiéndose su valoración a las 24 y 48 horas, donde 


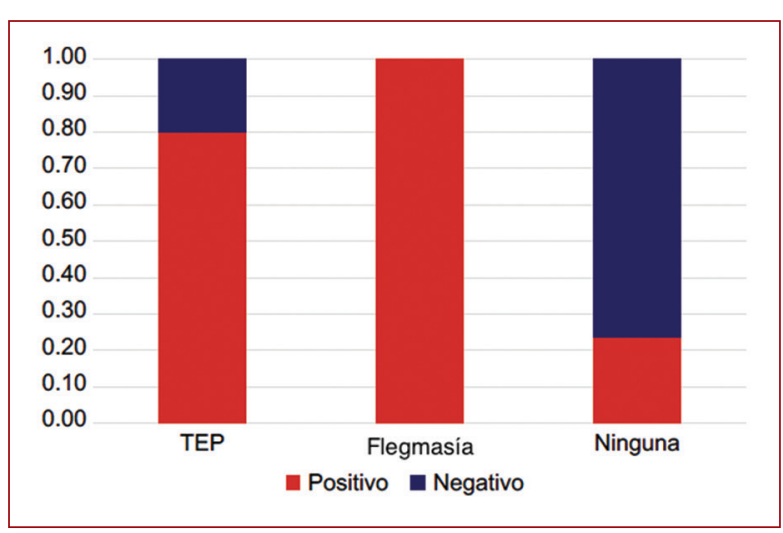

Figura 6. Complicaciones asociadas a TVP. TEP: tromboembolia pulmonar.

confirmamos el diagnóstico. Es posible que ocurran más episodios de TVP durante toda la hospitalización, sin embargo nuestra valoración depende de la solicitud de interconsulta por parte del médico tratante. Aunque al menos el $87 \%$ de los pacientes necesitaba un examen ultrasonográfico en serie, no siempre estaba disponible debido a recursos médicos limitados ${ }^{21-23}$.

En particular, la ubicación de la trombosis venosa fue principalmente encontrada en las venas proximales (íleo femoral) de miembro pélvico izquierdo ${ }^{24,25}$.

Los análisis multivariantes mostraron que un alto nivel de dímero $D$ se asocia como factor de riesgo para TVP, describiendo la fuerte asociación entre niveles altos de dímero $D$ y TVP ${ }^{25-27}$. Esto puede haber sido atribuido a un síndrome de respuesta inflamatoria sistémica para la activación de coagulación sanguínea, detectada con alto contenido de fibrinógeno ${ }^{26,27}$.

En nuestro estudio incluimos casos confirmados a COVID-19, referidos de otra unidad hospitalaria, pacientes que habían recibido algún otro tipo de tratamiento por trombosis venosa, ya fuera médico o quirúrgico. Los pacientes incluidos fueron a solicitud de interconsulta de servicios como unidad de urgencias, triaje respiratorio, hospitalizados en unidades de cuidados intensivos (UCl) y diferentes servicios del HGM.

Los factores de riesgo de TVP pueden ser transitorios o persistentes, aumentando el riesgo de tromboembolia venosa al inducir hipercoagulabilidad, estasis, daño o disfunción de la pared vascular ${ }^{27,28}$. El diagnóstico se puede excluir con seguridad si los niveles de dímero $\mathrm{D}$ son normales.

Además, la inmovilización y el cáncer son factores de riesgo para comorbilidades. Sin embargo, la comorbilidad no se asoció con TVP como factor de riesgo en nuestro estudio.
Informes anteriores han revelado que los pacientes con COVID-19 pueden rápidamente desarrollar enfermedades críticas, causando un alto nivel de respuesta inflamatoria, insuficiencia renal, insuficiencia respiratoria y disfunción hepática ${ }^{28,29}$.

La malignidad es el factor desencadenante más común de las flegmasías y está presente en aproximadamente el 20 al $40 \%$ de los pacientes. Aunque es poco común, es importante identificar a los pacientes con TVP iliofemorales masivas acompañadas de trombosis venosa superficial, ya que puede producir flegmasía cerúlea dolens, aumentando la presión venosa hasta el punto en el que la perfusión arterial de las extremidades se ve afectada. La mayoría de los casos están precedidos por flegmasía alba dolens, con síntomas de edema, dolor y palidez (alba) sin cianosis. A medida que avanza, el secuestro masivo de líquido puede conducir a la formación de flictenas y ampollas y finalmente sobreviene cianosis (cerúlea) y gangrena venosa, de modo que con frecuencia se produce colapso circulatorio y shock ${ }^{29,30}$.

Nuestra preferencia por la heparina no fraccionada IV como anticoagulante inicial se basa en la experiencia clínica y la falta de datos que respalden la heparina de bajo peso molecular o los anticoagulantes orales directos en esta población, así como la posible necesidad de interrumpir de forma aguda la anticoagulación, cuando se toma la decisión de proceder con la trombólisis y/o trombectomía. La trombólisis dirigida por catéter o la eliminación rápida del trombo oclusivo mediante técnicas manuales (trombectomía quirúrgica 0 dirigida por catéter) debe considerarse seriamente en esta población de pacientes ${ }^{30,31}$.

En nuestro estudio pudimos observar un alto porcentaje en sexo femenino, $61.4 \%$, y el $6 \%$ de TEP en los pacientes incluidos en nuestro estudio permanecieron ingresados en $\mathrm{UCl}$, asociado al deterioro de sus condiciones generales. Sin embargo, en este estudio los mecanismos que conducen a la TVP entre los pacientes con COVID-19 y la estancia en UCI pueden ser diferentes ${ }^{32}$.

Por tanto, se necesitan más estudios de tipo prospectivo en nuestra población, que nos permitan determinar los mecanismos involucrados en la enfermedad tromboembólica de utilidad para el manejo intrahospitalario y extrahospitalario de estos pacientes.

\section{Conclusión}

Los pacientes con COVID-19 presentan con frecuencia factores de riesgo trombótico, en su mayoría leucemias u otro tipo de cáncer; de igual forma la interacción 
de distintos factores de riesgo como la inmovilidad y obesidad predisponen a la estasis venosa $e$ hipercoagulabilidad condicionando a TVP y/o TEP, por lo que debemos establecer una profilaxis antitrombótica temprana en este grupo de pacientes.

La tasa de complicaciones en este grupo de pacientes está asociada con las comorbilidades, factores de riesgo y síndrome de respuesta inflamatoria sistémica que presenta nuestra población.

\section{Financiamiento}

La presente investigación no ha recibido ninguna beca específica de agencias de los sectores públicos, comercial o con ánimo de lucro.

\section{Conflicto de intereses}

Los autores declaran no tener conflicto de intereses.

\section{Responsabilidades éticas}

Protección de personas y animales. Los autores declaran que para esta investigación no se han realizado experimentos en seres humanos ni en animales.

Confidencialidad de los datos. Los autores declaran que han seguido los protocolos de su centro de trabajo sobre la publicación de datos de pacientes.

Derecho a la privacidad y consentimiento informado. Los autores han obtenido el consentimiento informado de los pacientes y/o sujetos referidos en el artículo. Este documento obra en poder del autor de correspondencia.

\section{Bibliografía}

1. World Health Organization. Coronavirus disease (COVID-19). Situation report 192 [Internet]. World Health Organization; 2020 [acceso: 31 de julio de 2020]. Disponible en: https www.who.int/docs/default source/coronaviruse/ situation reports/20200730 COVID 19 sitrep 192.pdf?sfvrsn × 5e52901f_4

2. Arachchillage DRJ, Laffan M. Abnormal coagulation parameters are associated with poor prognosis in patients with novel coronavirus pneumonia. J Thromb Haemost. 2020;18(5):1233-4.

3. Walters KA, D'Agnillo F, Sheng ZM, Kindrachuk J, Schwartzman LM, Kuestner RE, et al. 1918 pandemic influenza virus and Streptococcus pneumoniae co infection results in activation of coagulation and widespread pulmonary thrombosis in mice and humans. J Pathol. 2016;238(1):85-97.

4. Wang T, Chen R, Liu C, Liang W, Guan W, Tang R, et al. Attention should be paid to venous thromboembolism prophylaxis in the management of COVID-19. Lancet Haematol. 2020;7(5):e362-e363.

5. Raskob GE, Angchaisuksiri P, Blanco AN, Buller H, Gallus A, Hunt BJ, et al. Thrombosis: a major contributor to global disease burden. Arterioscler Thromb Vasc Biol. 2014;34(11):2363-71.

6. Deitelzweig SB, Lin J, Johnson BH, Schulman KL. Venous thromboembolism in the US: does race matter? J Thromb Thrombolysis. 2011;31(2):133-8.

7. Heit JA. Epidemiology of venous thromboembolism. Nat Rev Cardiol. 2015;12(8):464-74.

8. Criqui MH, Fronek A, Barrett-Connor E, Klauber MR, Gabriel S, Goodman D. The prevalence of peripheral arterial disease in a defined population. Circulation. 1985;71(3):510-5.
9. Hemingway JF, Singh N, Starnes BW. Emerging practice patterns in vascular surgery during the COVID-19 pandemic. J Vasc Surg. 2020;72(2):396-402.

10. Ünal EU, Mavioğlu HL, İscan HZ. Vascular surgery in the COVID-19 pandemic. J Vasc Surg. 2020;72(2):752-4

11. Sharma D, Rasmussen M, Han R, Whalin MK, Davis M, Kofke WA, et al. Anesthetic management of endovascular treatment of acute ischemic stroke during COVID-19 pandemic: Consensus Statement From Society for Neuroscience in Anesthesiology \& Critical Care (SNACC): Endorsed by Society of Vascular \& Interventional Neurology (SVIN), Society of Neurolnterventional Surgery (SNIS), Neurocritical Care Society (NCS), European Society of Minimally Invasive Neurological Therapy (ESMINT) and American Association of Neurological Surgeons (AANS) and Congress of Neurological Surgeons (CNS) Cerebrovascular Section. J Neurosurg Anesthesiol. 2020;32(3):193-201.

12. Khider L, Gendron N, Goudot G, Chocron R, HauwखBerlemont C, Cheng $\mathrm{C}$, et al. Curative anticoagulation prevents endothelial lesion in COVID-19 patients. J Thromb Haemost. 2020;18(9):2391-9.

13. Di Martino M, García Septiem J, Maqueda González R, Muñoz de Nova JL, de la Hoz Rodríguez A, Correa Bonito A, et al. Cirugía electiva durante la pandemia por SARS-CoV-2 (COVID-19): análisis de morbimortalidad y recomendaciones sobre priorización de los pacientes y medidas de seguridad. Cir Esp. 2020;98(9):525-32.

14. Cabrera-Vargas LF, Pedraza Ciro M, Torregrosa Almonacid L, Figueredo E. Cirugía durante la pandemia del SARS-CoV-2/COVID-19: el efecto de la generación de aerosoles de partículas en escenarios quirúrgicos. Rev Colomb Cir. 2020;35(2):190-9.

15. Artinyan A, Orcutt ST, Anaya DA, Richardson P, Chen GJ, Berger DH Infectious postoperative complications decrease long-term survival in patients undergoing curative surgery for colorectal cancer: A study of 12,075 patients. Ann Surg. 2015;261(3):497-505

16. Gomez-Arbelaez D, Ibarra-Sanchez G, Garcia-Gutierrez A, Comanges-Yeboles A, Ansuategui-Vicente M, Gonzalez-Fajardo JA. COVID-19-related aortic thrombosis: A report of four cases. Ann Vasc Surg. 2020;67:10-3.

17. Spiezia L, Boscolo A, Poletto F, Cerruti L, Tiberio I, Campello E, et al. COVID-19-related severe hypercoagulability in patients admitted to intensive care unit for acute respiratory failure. Thromb Haemost. 2020;120(06):998-1000.

18. Mucha SR, Dugar S, McCrae K, Joseph D, Bartholomew J, Sacha GL, et al. Coagulopathy in COVID-19: Manifestations and management. Cleve Clin J Med. 2020;87(8):461-8.

19. Madjid M, Safavi-Naeini $P$, Solomon SD, Vardeny O. Potential effects of coronaviruses on the cardiovascular system: A review. JAMA Cardiol. 2020;5(7):831.

20. Wang D, Hu B, Hu C, Zhu F, Liu X, Zhang J, et al. Clinical characteristics of 138 hospitalized patients with 2019 novel coronavirus-infected pneumonia in Wuhan, China. JAMA. 2020;323(11):1061.

21. Wichmann D, Sperhake J-P, Lütgehetmann M, Steurer S, Edler C, Heinemann $\mathrm{A}$, et al. Autopsy findings and venous thromboembolism in patients with COVID-19: A prospective cohort study. Ann Intern Med. 2020;173(4):268-77.

22. Verdoni L, Mazza A, Gervasoni A, Martelli L, Ruggeri M, Ciuffreda M, et al. An outbreak of severe Kawasaki-like disease at the Italian epicentre of the SARS-CoV-2 epidemic: an observational cohort study. Lancet. 2020;395(10239):1771-8.

23. Teuwen L-A, Geldhof V, Pasut A, Carmeliet P. COVID-19: the vasculature unleashed. Nat Rev Immunol. 2020;20(7):389-91.

24. Mangalmurti N, Hunter CA. Cytokine storms: Understanding COVID-19. Immunity. 2020;53(1):19-25.

25. Magro C, Mulvey JJ, Berlin D, Nuovo G, Salvatore S, Harp J, et al. Complement associated microvascular injury and thrombosis in the pathogenesis of severe COVID-19 infection: A report of five cases. Transl Res. 2020;220:1-13.

26. Li K, Hao Z, Zhao X, Du J, Zhou Y SARS-CoV-2 infection-induced immune responses: Friends or foes? Scand J Immunol 2020;92(2):e12895.

27. Iba T, Connors JM, Levy JH. The coagulopathy, endotheliopathy, and vasculitis of COVID-19. Inflamm Res. 2020;69(12):1181-9.

28. Huisman A, Beun R, Sikma M, Westerink J, Kusadasi N. Involvement of ADAMTS13 and von Willebrand factor in thromboembolic events in patients infected with SARS-CoV-2. Int J Lab Hem. 2020;42(5):e211-e212.

29. Costela-Ruiz VJ, Illescas-Montes R, Puerta-Puerta JM, Ruiz C, Melguizo-Rodríguez L. SARS-CoV-2 infection: The role of cytokines in COVID-19 disease. Cytokine Growth Factor Rev. 2020;54:62-75.

30. Oguzkurt L, Ozkan U, Demirturk OS, Gur S. Endovascular treatment of phlegmasia cerulea dolens with impending venous gangrene: Manual aspiration thrombectomy as the first-line thrombus removal method. Cardiovasc Intervent Radiol. 2011;34(6):1214-21.

31. Hu H, Cai Y, Wang C, Yang C, Duan Z, Zhang J, et al. Successful treatment of posttraumatic phlegmasia cerulea dolens by reconstructing the external iliac vein: a case report. J Med Case Reports. 2014;8(1):149.

32. Jayarangaiah A, Kariyanna PT, Chen X, Jayarangaiah A, Kumar A COVID-19-associated coagulopathy: An exacerbated immunothrombosis response. Clin Appl Thromb Hemost. 2020;26:107602962094329. 